\title{
Extremely short diapause in rotifers and its fitness consequences
}

\author{
Claus-Peter Stelzer (i)
}

Received: 13 November 2015/Revised: 31 July 2016/ Accepted: 31 July 2016/Published online: 17 August 2016

(C) The Author(s) 2016. This article is published with open access at Springerlink.com

\begin{abstract}
All monogonont rotifers have a diapause stage (=resting egg, RE), which allows them to endure unfavourable periods and to disperse across nonaquatic boundaries. However, recent research has shown that REs may often develop spontaneously within a few days, which seems to partly offset these adaptive explanations. In this study, I determined the minimum duration of RE development in a $\mathrm{Bra}$ chionus calyciflorus Pallas 1766 strain in relation to other life-history variables. RE development took at least $72 \mathrm{~h}$, but $50 \%$ of those REs that hatched within the first week did so in a much synchronized manner, within 72-82 h. By contrast, amictic egg development took $11 \mathrm{~h}$, while REs that had been previously stored in the cold/dark for weeks, hatched within $21 \mathrm{~h}$ if exposed to warm/light conditions. I discuss the fitness consequences of such fast RE development and whether it can ameliorate the costs of sex in this system. I also propose a conceptual model of RE
\end{abstract}

Guest editors: M. Devetter, D. Fontaneto, C. D. Jersabek, D. B. Mark Welch, L. May \& E. J. Walsh / Evolving rotifers, evolving science

Electronic supplementary material The online version of this article (doi:10.1007/s10750-016-2937-x) contains supplementary material, which is available to authorized users.

C.-P. Stelzer $(\bowtie)$

Research Institute for Limnology, University of Innsbruck, Mondseestr. 9, 5310 Mondsee, Austria e-mail: claus-peter.stelzer@uibk.ac.at development, which assumes two obligatory phases of pre- and post-diapause development and a facultative phase of dormancy. The latter phase may be missing in spontaneously developing REs.

Keywords Resting egg bank - Brachionus .

Dormancy $\cdot$ Embryonic development

\section{Introduction}

Many aquatic invertebrates possess a diapause stage that allows them to endure extended periods of unfavourable conditions and to disperse passively across non-aquatic boundaries (Hairston, 1996; Brendonck \& De Meester, 2003). This diapause stage is often linked to sexual recombination. For example, organisms with heterogonic life cycles (e.g. Daphnia or monogonont rotifers) reproduce asexually for several generations by producing subitaneous eggs, which hatch within hours, whereas once a zygote has been formed after sexual reproduction, the embryo arrests its development and enters diapause (=resting egg, RE). Resumption of development in REs is thought to involve a combination of external triggers (usually conditions indicating that environmental conditions are favourable again), but there may also be a period when the development is obligatorily blocked (e.g. Brendonck, 1996). The duration of diapause is typically considered long relative to the lifespan of active individuals. That is, diapause lasts 
for months to years, while individual lifespan is only days to a few weeks (Gilbert, 1974; Pourriot \& Snell, 1983; Schröder, 2005).

The short-term fitness consequences of such life cycles are well established: high rates of sex may decrease the population growth rate through a component of male production ("cost of males") and a component of diapause, since the hatchlings of REs do not contribute to a growing population (Serra \& Snell, 2009; Stelzer, 2011). Over short time scales, this can lead to situations where facultative sexuals are being outcompeted by obligate parthenogenesis, if the individuals in the initial population differed in their propensity for sex (e.g. Stelzer, 2011). In nature, Carmona et al. (2009) observed that selection against high propensity for sex may occur during a growing season. Yet successful invasions, i.e. those with complete replacement of sexuals, seem to be rare and it is thus of importance to know the forces that prevent them from happening. To some extent, this apparent contradiction may be resolved by simply increasing the time scale, i.e. geometric fitness of asexuals should become zero in the long run as the chance of a catastrophic event increases, because they lack the (sexual) diapause stage. However, there are indications that the actual problem is more complex. Indeed, the tight link between diapause and sexual reproduction has been challenged by many empirical studies, even for those organisms that traditionally served as a paradigm for such a link (Stelzer, 2015).

For example, in rotifers several empirical studies have established that "diapausing eggs are not always sexual" and that "sexual eggs are not always diapausing". First, the discovery of pseudosexual eggs has shown that some species or strains are able to asexually produce diapausing eggs (several cases cited in Schröder, 2005). For example, the rotifer Synchaeta pectinata Ehrenberg 1832 may produce thick-shelled asexual eggs that undergo an obligatory period of dormancy, which lasts for 14 days (Gilbert, 1995). Since the production of these eggs is triggered by food deprivation, it is viewed as an adaptation that enables individual clones to survive extended periods of starvation, when normal clonal growth would not be an option (Gilbert \& Schreiber, 1998). Second, evidence has accumulated of cases where diapause is extremely short, lasting only a few days. Cases of extremely short diapause are best known in rotifers of the genus Brachionus. For example, several studies have documented that $>50 \%$ of all REs may hatch within the first week after they had been produced (Gilbert, 2001; Gilbert \& Dieguez, 2010; Scheuerl \& Stelzer, 2013; Martínez-Ruiz \& García-Roger, 2015), and studies of experimental evolution sometimes mention this phenomenon as a side observation (e.g. Becks \& Agrawal, 2010).

Extremely short diapause should lead to higher short-term fitness, compared to long diapause, because hatchlings from REs can actually contribute to a growing population. However, since much of the evidence for extremely short diapause is either anecdotal or semi-quantitative, it is difficult to quantify its effect on the population growth rate. Important open questions are as follows: Is there a minimum diapause duration, below which development cannot be shortened? How quick is the development of diapausing eggs relative to amictic eggs (AE)? What are the consequences of short diapause for population growth, i.e. after accounting for other fitness components, such as juvenile development and survival and fecundity schedules of adults? In this study, I address these questions and obtain precise estimates of minimum diapause duration in a $B$. calyciflorus strain using time-lapse recording of egg development (REs vs. AEs). I will relate these data to the full life cycle, by estimating the duration of juvenile development and survival and fecundity schedules in the same strain of B. calyciflorus. Finally, using these data, I will estimate the consequences of extremely short diapause duration on the population growth rate.

\section{Methods}

Animals and culturing procedures

A multiclonal strain of B. calyciflorus was established from Egelsee, a shallow but permanently filled pond in the vicinity of Salzburg Austria. The ancestors of this strain have been used in a previous publication ("Salt population4" in Scheuerl \& Stelzer, 2013). Originally, this population was founded by 112 rotifer clones, and it was subsequently kept under laboratory selection for several months with frequent episodes of sexual reproduction (Scheuerl \& Stelzer, 2013). Rotifers were cultured in COMBO medium (Kilham et al., 1998) with Chlamydomonas reinhardtii Dangeard 1888 as food, usually at concentrations of 400,000 
cells $\mathrm{ml}^{-1}$. Ambient light conditions during the experiments were $\sim 150 \mu \mathrm{mol}$ quanta $\mathrm{m}^{2} \mathrm{~s}^{-1}$. Temperature was set to $23.5^{\circ} \mathrm{C}$ in temperature-controlled rooms and incubators.

\section{Measurements of embryonic development time}

Embryonic development time was measured by two methods, (i) a conventional method in which hatching was controlled at $12 \mathrm{~h}$ intervals and (ii) an automated method, which involved time-lapse recording of up to 96 eggs at $30 \mathrm{~min}$ intervals. In both cases, egg cohorts were generated by harvesting egg bearing females from growing mass cultures ( 11 volume). Eggs were stripped from these females by vigorous vortexing. Then the females without eggs were placed into small glass containers with about $2 \mathrm{~mL}$ food suspension. The production of new eggs was checked in regular intervals ( $4 \mathrm{~h}$ for the conventional method; $30 \mathrm{~min}$ for time-lapse recording), and females with newly attached eggs were isolated from the others. Subsequently, each single egg was removed from each female using a capillary with a $125 \mu \mathrm{m}$ opening (Mid Atlantic Stripper tips ${ }^{\circledR}$ by Origio). Eggs were individually placed into wells of a 96-well plate with a black rim and a clear bottom (Greiner Bio-One, no. 655096), filled with $400 \mu \mathrm{l}$ fresh COMBO Medium. Prior to time-lapse recording of hatching times, $18 \times 18 \mathrm{~mm}$ coverslips were placed onto the 96-well plate (one coverslip covering four adjacent wells) to prevent evaporation during the recording.

The time-lapse recording system consisted of a relatively simple custom-made microscope, a motorized scanning table (Zaber ${ }^{\circledR}$ ASR100B120B), and a computer, which were all placed into an incubator set to $23.5^{\circ} \mathrm{C}$ temperature. The scanning table can hold a 96-well plate and allows precise movement control such that each individual well can be selected at $\mu \mathrm{m}$ precision. The optics of the system resembled an inverted microscope and consisted of a $1 \times$ lens, a 90-mm optical tube, and a 6.6 MP digital camera (PixeLINK $^{\circledR}$ PL-B781F), which were all mounted as a unit on an optical breadboard (Thorlabs Inc.). The motorized scanning table was mounted above the optical system using standard optomechanical components, such as optical posts and mounting brackets (Thorlabs Inc.). The 96-well plate was illuminated from above by two fluorescent daylight bulbs at a light intensity of $150 \mu \mathrm{mol}$ quanta $\mathrm{m}^{2} \mathrm{~s}^{-1}$. An additional fan inside the incubator ensured equal temperature distribution, especially around the scanning table area. Both the scanning table and the digital camera were controlled by the computer with a self-written Labview ${ }^{\circledR}$ program. Pictures were stored automatically on the computer, with time stamps and separately for each well, and were later evaluated to determine the time interval of hatching.

Effect of experimental treatments on embryonic development time

Two experiments were conducted for quantifying embryonic development time of REs in B. calyciflorus. In the first experiment, I examined the effect of the collection procedure on synchronization of RE hatching. REs were either (i) collected from the bottom of a growing B. calyciflorus culture, which was inoculated 4 days before, or (ii) stripped from actively swimming females (i.e. females carrying 1-2 REs), or (iii) their production age has been narrowed to a $9 \mathrm{~h}$ interval, using the stripping-reattachment procedure described above. All three RE groups were subsequently checked for hatching in $12 \mathrm{~h}$ intervals for a duration of 7 days. In the second experiment, I used time-lapse recording for obtaining more precise estimates of the hatching times and to compare the hatching times of three types of eggs: (1) REs kept under high light intensity all the time $(150 \mu \mathrm{mol}$ quanta $\mathrm{m}^{2} \mathrm{~s}^{-1}$ ), (2) AEs kept under the same conditions, (3) REs, which were stored at cold/dark conditions $\left(5^{\circ} \mathrm{C}\right)$ for 2 weeks immediately after they were produced, and then incubated in high light intensity. In the second experiment, egg production age was narrowed down to a 30 min interval and hatching of all egg types was checked in $30 \mathrm{~min}$ intervals for up to $300 \mathrm{~h}$.

\section{Life table experiment}

A life table experiment was conducted to establish the contribution of hatchlings from REs to population growth, i.e. the lifetime-fecundity schedule of individuals that only recruit via hatching of REs (vs. individuals that reproduce asexually via AEs). Cohorts were initiated from $4 \mathrm{~h}$ cohorts of AEs as described above. These AEs were isolated from a population at high population density, so the individuals that were hatching from these eggs were either amictic females 
or (fertilized) mictic females. Individuals of both groups were transferred to new plates with fresh food suspension. Survival and egg/offspring production were recorded during each transfer. Resting eggs were collected into separate wells and were subsequently kept under the same light and temperature conditions until they hatched. Hatching of REs was checked at $12 \mathrm{~h}$ intervals. In addition, the duration of juvenile development was measured in for amictic and REproducing females. For these measurements, the control interval for extrusion of the first egg was decreased to $4 \mathrm{~h}$.

Life tables were calculated from the reconstructed lifetime-fecundity schedules of AE-producing and RE-producing females. In these calculations, the life cycle was considered starting with the zygote (egg), and the age at first reproduction was defined as the extrusion of the first egg by a mother. Thus, the life cycle was composed of the following sequence of periods: embryonic development, juvenile phase and adult phase. In principle, this is equivalent to another method commonly used in constructing rotifer life tables, in which life starts with hatching of the mother and reproduction starts with hatching of her first offspring. However, the method used in the present paper is more adaptable to life cycle variation in terms of the embryonic development time, because reproduction is simply delayed by the additional time the mother requires for her own embryonic development. This method also avoids complications that would arise in the calculation of $\mathrm{mx}$-values (for example, in RE producers, most of the mothers would already be dead by the time their offspring hatches). For REproducing females, the method used in the present paper is very straightforward since the production of REs can be easily quantified, because they detach from females after 1-2 days. For amictic females, the following adjustment was necessary: I recorded the offspring as hatchlings and later adjusted the fecundity values by shifting each value to the preceding $12 \mathrm{~h}$ observation point (corresponding to their approximate time of egg extrusion). This was possible because the embryonic development time of AEs was roughly identical with one observation interval $(\sim 11 \mathrm{~h}$, see results).

Hypothetical population growth rates of RE-producing and AE-producing females were calculated from the life table data. The purpose of these calculations was to obtain an estimate of population growth that could be accomplished by a hypothetical 'sexually reproducing rotifer with short diapause', which marks the opposite extreme to an 'obligate parthenogen'. Three important simplifications were made as follows: (i) It was assumed that each RE hatches exactly 3 days after it had been produced (i.e. there was no RE mortality and all REs were shortdiapausing); (ii) Females hatching from REs were again mictic. Thus, this idealized life table for RE producers refers to a hypothetical life cycle of a monogonont rotifer where the asexual cycle has been "shorted-out". While this condition might be very rare in natural systems, there is at least no fundamental biological constraint preventing it, since mictic hatchlings from REs have been found in a population of Hexarthra sp. (Schröder et al., 2007); (iii) The fecundity of RE-producing females was adjusted to account for sex ratio allocation in the population. According to previous studies (Aparici et al., 1998, 2002), monogonont rotifers are expected to have an equal frequency of male-producing and resting egg-producing females. Thus, fecundities of RE-producing females were adjusted by multiplying them with the factor 0.5 (because male producers do not contribute to population growth). The intrinsic rate of increase $r$ was then calculated by iteratively solving the Euler-Lotka equation

$\sum e^{-r x} \cdot l_{x} \cdot m_{x}=1$,

where $l_{x}$ is the survivorship until age $x$ and $m_{x}$ is the mean number of eggs of a female at age $x$. Confidence intervals $(95 \%)$ for the intrinsic rate of increase were estimated for 1000 bootstrapped replicates with MATLAB $^{\circledR}$ software, using the command 'bootci'.

\section{Results}

In the Brachionus strain of this study, a considerable proportion of REs hatched soon after they had been produced. For example, REs collected from the bottom of a growing culture, which was inoculated 4 days before RE collection, exhibited a linear hatching pattern over time, with a more or less constant fraction of $22 \%$ hatchlings per day until $98 \%$ of all REs had hatched (Fig. 1). By contrast, the hatching curve got more S-shaped with increasing synchronization of egg production age, as the "lag phase" (the 


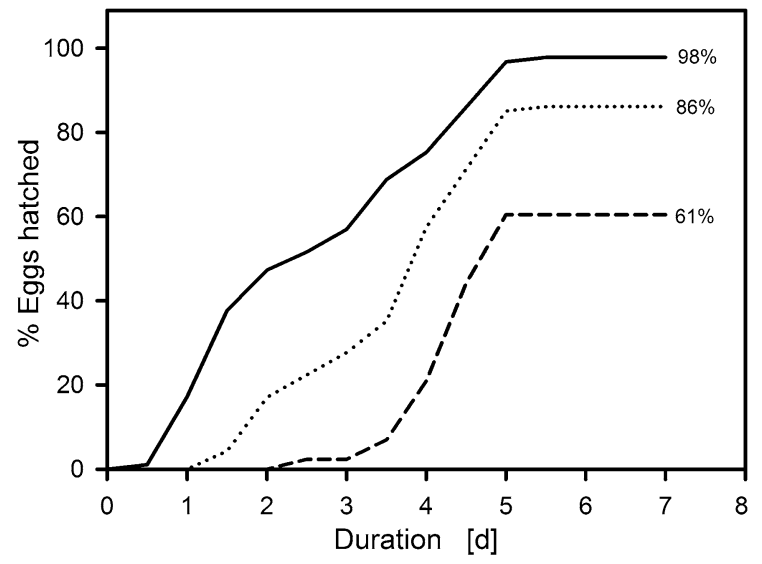

Fig. 1 Cumulative proportions of hatched resting eggs. Solid line: eggs collected from the bottom of a culture; dotted line: eggs stripped from females, which were carrying two REs; dashed line: eggs produced by females within a $9 \mathrm{~h}$ interval. The percentages at the end of each line indicate the proportion of resting eggs that hatched after 7 days

time until the first hatchlings appear) increased. Since there were virtually no new hatchlings after 5 days in all egg collection methods, the steepness of the hatching curve also increased with increasing synchronization, with $25 \%$ hatchlings per day if eggs were directly stripped from females (dotted line in Fig. 1) and $30 \%$ hatchlings per day (dashed line in Fig. 1) if the eggs were produced within a $9 \mathrm{~h}$ interval. The cumulative proportions of hatchlings in all egg collection methods approached a plateau by day five.

To get a more precise estimate of the minimum time required for RE hatching, I used a time-lapse recording system in which RE production was narrowed down to 30 min intervals and hatching was monitored in $30 \mathrm{~min}$ intervals (for up to 7 days). Under such conditions, the first hatchling appeared after $72 \mathrm{~h}$ (Fig. 2), which was followed by $\sim 15 \%$ of all REs that hatched within a relatively short time period afterwards (72-82 h, median: $76 \mathrm{~h}$ ). After this first wave of hatchlings, additional hatchlings appeared in a more unsynchronized manner. Since the total percentage of hatchlings was only $31 \%$ (up to and including $300 \mathrm{~h}$ ), the fraction that hatched within $72-82 \mathrm{~h}$ amounted to almost $50 \%$ of all hatchlings. The REs that did not hatch until $300 \mathrm{~h}$ still looked viable according to the morphological classification of Garcia-Roger et al. (2005), i.e. their multinuclear embryo filled more than $75 \%$ of maximum embryo volume. Two additional treatments were used to compare the

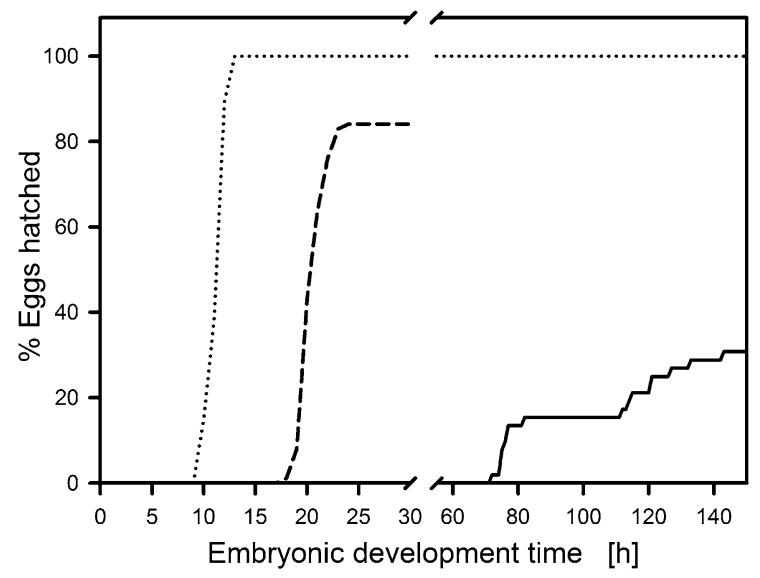

Fig. 2 Cumulative proportions of hatched eggs using timelapse recording. All eggs were produced within a 30-min interval and were checked for hatching in $30 \mathrm{~min}$ intervals. Treatments: AEs (dotted line); resting eggs stored for 3 weeks at $7{ }^{\circ} \mathrm{C}$ in the dark until exposure to hatching conditions (dashed line); resting eggs stripped from females and directly exposed to the hatching conditions (solid line)

length of embryonic development times: AEs and REs stored in cold/dark. Both treatments were run under exactly the same light and temperature conditions. In AEs, all offspring hatched within 10-13 h (median: $11 \mathrm{~h}$ ) after the eggs had been produced (Fig. 2, dotted line). By contrast, REs that had been stored at cold/dark conditions took longer time to develop (19-23 h). In both treatments, hatching was strongly synchronized.

A life table experiment was used to compare fitnessrelated traits other than just embryonic development time. Overall, the lifetime-fecundity schedules differed considerably between AE- and RE-producing females (Fig. 3; supplementary data). RE producers took a slightly longer time to reach adulthood, i.e. the time between mother hatching and extrusion of her first egg, was longer $(26.7 \pm 2.4$ vs. $20.6 \pm 2.9$ h). This difference was statistically significant (Students $t$ test, $t=8.23$, d.f. $=50, P<0.001)$. In addition, total egg production during adult life was significantly higher in AE producers than in RE producers $(16.1 \pm 0.9$ vs. $6.2 \pm 0.3$ eggs per female), which was also statistically significant (Kruskal-Wallis test, $H=35.89$, d.f. $=1, P<0.001)$. However, the survival curves did not differ noticeably. Thus, the above-mentioned difference in fecundity between the two reproductive types can be mainly attributed to longer egg laying intervals in RE producers: on average, the egg laying 


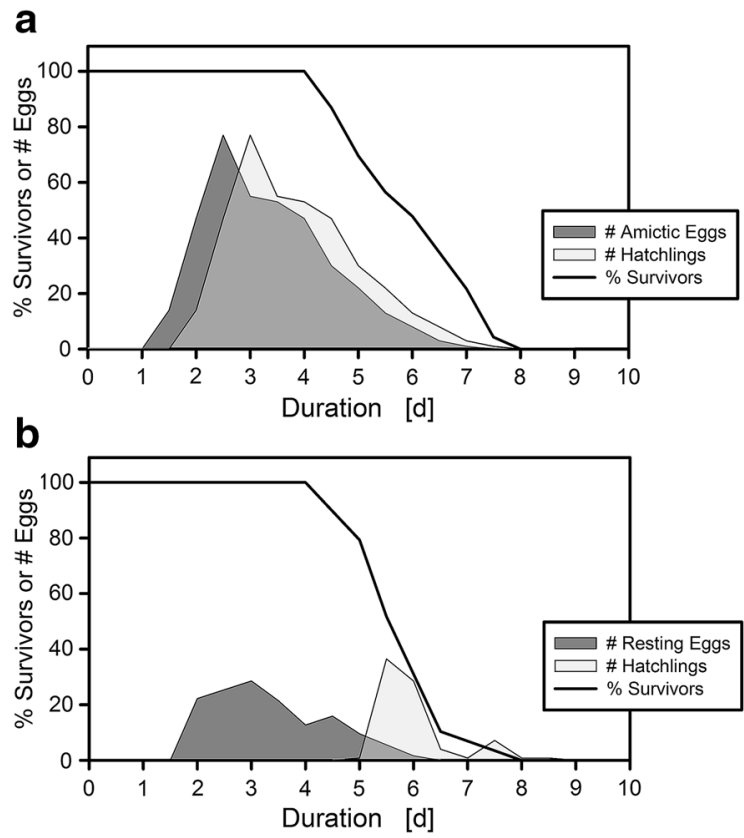

Fig. 3 Survival/fecundity schedules of AE-producing females (a) versus RE-producing females (b). Fecundity data are displayed as absolute numbers of eggs (dark grey) and as the number of hatchlings (light grey) per 12-h time interval. Egg and hatchling numbers have been scaled to an initial cohort size of 23 females in both cases. Duration refers to the age of the mothers since hatching

interval was $12.3 \pm 0.3 \mathrm{~h}$ in $\mathrm{RE}$ producers vs. $5.8 \pm 0.2 \mathrm{~h}$ in AE producers. In the life table experiment, $55 \%$ of all REs hatched within 3-6 days and the first hatchlings of REs were found $72 \mathrm{~h}$ after the first interval when RE production had been recorded (Fig. 3b). Hypothetical population growth rates of RE-producing and AE-producing females were 0.181 (95\% confidence intervals 0.057 and $0.280 \mathrm{~d}^{-1}$ ) and $0.813 \mathrm{~d}^{-1}$ (95\% confidence intervals 0.563 and 1.066 $\left.\mathrm{d}^{-1}\right)$, respectively.

\section{Discussion}

In this study, I found that high proportions of the REs produced by a $B$. calyciflorus strain hatched within few days after they had been produced, which is in agreement with several earlier studies (Gilbert, 2001; Gilbert \& Dieguez, 2010; Scheuerl \& Stelzer, 2013; Martínez-Ruiz \& García-Roger, 2015). Precise measurements of RE hatching times, using time-lapse recording of the hatching process, showed that no RE hatched before $72 \mathrm{~h}$, and that a large proportion of those eggs that hatched within the first week $(\sim 50 \%)$ did indeed hatch within a fairly short time interval (72-82 h). For comparison, under the same temperature and light conditions, embryonic development of AEs lasted only $11 \mathrm{~h}$, while REs that were stored at $7{ }^{\circ} \mathrm{C}$ in the dark for 3 weeks hatched within 19-23 h, after they had received the hatching stimulus (transfer to $23.5^{\circ} \mathrm{C}$ and $150 \mu \mathrm{mol}$ quanta $\mathrm{m}^{2} \mathrm{~s}^{-1}$ ). How do such observations fit into the current understanding of RE development?

Conceptual model on RE development

A simple conceptual model of RE development is shown in Fig. 4. According to this model, RE development comprises three phases, a pre-diapause phase, the actual diapause and a post-diapause phase. In the pre-diapause phase, developmental processes that allow the embryo to become dormant for long time periods are activated. Such processes may include karyogamy, the initial divisions and structural changes in the RE (Wurdak et al., 1978; Hagiwara et al., 1995; Boschetti et al., 2011). The second phase corresponds to the actual state of dormancy and its duration can be highly variable. During this phase, embryonic development is believed to be arrested at a stage of 30-60 cells (Boschetti et al., 2011). On the other hand, this phase is characterized by transcriptional activity, due to activation of pathways and biochemical processes that allow the embryo to withstand prolonged times of dormancy and desiccation (Denekamp et al., $2009,2011)$. The transition to phase 3 , post-diapause development, is thought to involve a variety of exogeneous triggers and endogeneous variables (Pourriot \& Snell, 1983; Schröder, 2005). Exogeneous triggers may be related to environmental conditions indicating that the habitat becomes suitable after a period of adversity (e.g. rehydration, increases in temperature, light, salinity, or oxygen). Endogeneous variables with known effects on the duration of diapause are, e.g. the age of the mother, or genotype (Martínez-Ruiz \& García-Roger, 2015). The third phase, post-diapause development, consists of the embryonic development that ultimately results in a fully differentiated amictic hatchling. Like the prediapause phase, it is determined by obligatory developmental processes. In most species, hatchlings from REs are morphologically not different from the 


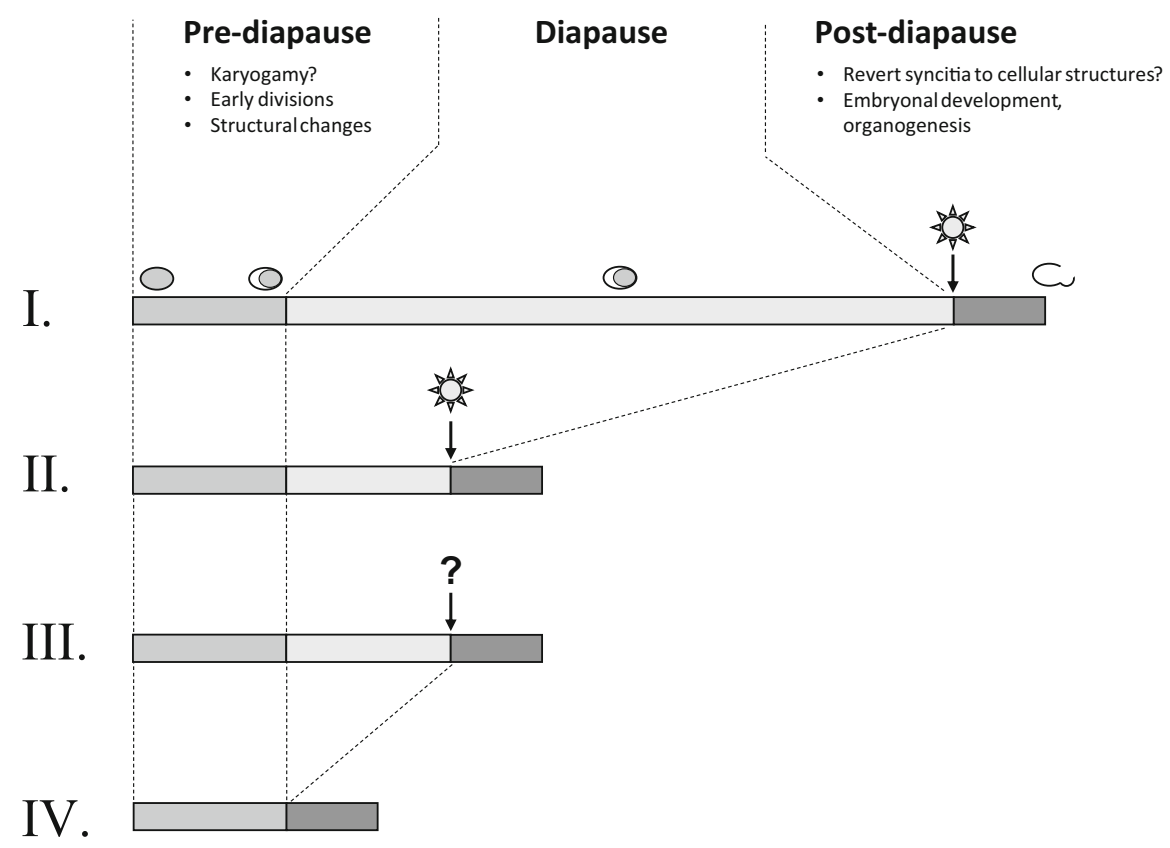

Fig. 4 A simple conceptual model for resting egg development. RE development is divided into three phases, prediapause, diapause and post-diapause. The duration of prediapause and post-diapause development is assumed to be constant in this model, while the actual diapause phase is highly variable $(I-I V$.). Furthermore, the triggers that end phase 2

hatchlings of AEs (some exceptions from this are reviewed by Gilbert \& Schroder, 2004). Thus, the developmental processes in late-stage REs might be similar to those in AEs.

How do the data reported in this study confer to such a model? The duration of post-diapause development (phase 3) can be inferred from the development time of REs that have been stored under cold and dark conditions, after they received the hatching cue (c.f., Fig. 2 solid line). Interestingly, post-diapause development lasted for $21 \mathrm{~h}$ in my experiments while the development of an AE took only $11 \mathrm{~h}$. As mentioned above, both egg types yield the same offspring (an amictic female). Moreover, AE development should include even more divisions, since it starts with cleavage, while the diapausing embryo typically consists of 30-60 nuclei when it resumes development (Hagiwara et al., 1995; Boschetti et al., 2011). A possible explanation for the delay is that RE embryos are in a developmental stage that cannot directly proceed with differentiation and organogenesis such as in an early AE, and that instead "diapausespecific" pathways have to be activated before normal (diapause) might be either external, environmental factors (I., II., indicated by the sun symbol), or unknown internal factors (III., indicated by the question mark). Finally, phase 2 (diapause) might be entirely missing in spontaneously developing resting eggs $(I V$.

embryonic development can resume. For example, it has been noted by several authors that diapausing embryos largely consist of syncitia, while embryos of AEs are of cellular structure (Gilbert, 1989; Boschetti et al., 2011). Thus, it is possible that the syncytia have to revert into cells before the reactivated RE embryo can resume normal development, which may take additional time.

The duration of pre-diapause development (phase 1) can be estimated from the data of this study, if we assume that spontaneously developing REs completely omit diapause (phase 2). Under this assumption, prediapause should last for $\sim 2$ days $(72-21=51 \mathrm{~h})$. Pre-diapause development is characterized by structural changes in the RE, such as changes in egg shell and inner lining, as well as the formation of an extraembryonic space (Wurdak et al., 1978). It is also characterized by early cell divisions until a stage that may resemble gastrulation (Boschetti et al., 2011). Possibly, the early pre-diapause development might even include the final steps of karyogamy, since it has been observed that the male nucleus stays physically separated from the oocyte nucleus until the very end of 
oocyte growth (Storch, 1924). In conclusion, the time required for pre-diapause development might depend on processes required for preparation of the diapausing stage, as well as time required for finishing sexual processes. The latter would be consistent with a cellular mechanical cost of meiosis, which has been suggested as a major cost of sex in unicellular organisms and organisms with short egg-to-egg cycles, such as rotifers (Lewis, 1983). Future studies need to elucidate the specific processes occurring during pre-diapause, such as metabolic, molecular, or cellular changes, in order to clarify whether a 2-day period is indeed necessary for their completion.

Diapause (phase 2): In my experiments, a cold and dark treatment could effectively "lock" embryos in diapause (since no egg stored under such conditions hatched within the 3-week period). In contrast, if such REs were kept under experimental conditions, a considerable fraction of the embryos spontaneously developed and hatched within 72-82 h. Spontaneous development in the absence of a cold $\&$ dark period seems to defy the notion that such a period is strictly necessary for RE hatching. An open question is if "early hatchers", such as the rotifer strain in this study, are an exceptional genetic variant in which the default phase 2 is "shorted-out", or whether they are actually quite common in natural populations. In the latter case, the duration of dormancy might be mainly determined by external factors that block RE hatching (e.g. desiccation, cold \& dark). Nevertheless, a considerable and variable fraction of REs in this study were not early hatchers, i.e. they did not hatch until the end of the 1 week duration of my experiments. Few of these eggs could be induced to hatch after several weeks and most of them remained dormant, but still looked viable (personal observation). This suggests that there may also be endogeneous mechanisms that lock RE embryos into diapause for variable durations, which are unrelated to environmental triggers indicating a favourable environment. Such mechanisms might be a component of a diversified bet-hedging strategy in the classical sense (Martínez-Ruiz \& García-Roger, 2015).

\section{Costs on population growth}

Resting egg production should result in large costs to population growth, since RE development, even if it is extremely fast, is considerably longer than AE development (72 vs. $11 \mathrm{~h}$ ). A previous model on the "cost of diapause" made the simplification of RE development being infinitely long (Stelzer, 2011), which is clearly not appropriate for the strain used in the present study. Thus, it may be suspected that spontaneous development of REs will to some extent ameliorate costs to population growth. In the present study, the estimated population growth rate of a hypothetical (obligate) sexual rotifer with a short 3-day diapause was $0.181 \mathrm{~d}^{-1}$. This value was much lower than the growth rate of amictic females $\left(0.813 \mathrm{~d}^{-1}\right)$. Nevertheless, it would allow considerable population growth on its own (i.e. it would allow a population to double every 4 days).

Several factors are responsible for the much lower population growth rate of this hypothetical sexual rotifer compared to its asexual counterpart: (1) longer egg development (72 vs. $11 \mathrm{~h}$ ), (2) slightly longer juvenile development, (3) reduced fecundity, due to longer egg intervals and lower lifetime egg production, (4) 'wasteful' production of males, which should lead to an additional $50 \%$ reduction in the reproductive output of mictic females. To summarize, even though extremely short diapause ameliorates the "cost of diapause" in this system, other "costs of sex" and demographic differences in the life history between RE-producing and amictic females should put a sexual rotifer at strong disadvantage compared to cyclical parthenogens with low sex propensity or an asexual mutant.

Normally, there is no "short-circuit" of the asexual cycle (i.e. mictic females directly hatching from REs) in monogonont rotifers (for an exception, see Schröder et al., 2007). A more likely scenario in nature is that cyclically parthenogenetic rotifers would vary in their sexual propensities and/or proportion of short- vs. long-diapausing eggs. It is important to understand and predict how such variants would compete against each other, or against obligate asexual mutants (Stelzer, 2015). The data reported in the present paper could be used for parametrizing a model of the full life cycle of a cyclical parthenogen (Stelzer \& Lehtonen, 2016), which allows analysing the contributions of the various sexual costs and/or to make better predictions on evolutionary stable strategies given various ecological settings (e.g. season length, habitat predictability).

In conclusion, rotifer strains exhibiting extremely short diapause (such as the strain used in this study) challenge the conventional view, which states that 
diapause in rotifers is typically long relative to lifetime of individuals. However, such observations are consistent with many other recent findings showing that life-history traits, especially those related to sexual reproduction, can be highly variable within and among populations (e.g. Campillo et al., 2009; Carmona et al., 2009). The causes and consequences of extremely short diapause in natural communities give rise to many unanswered questions. In which habitats is short diapause favoured? Is short diapause completely suppressed in deep lakes, where eggs sink to the cold, dark and anoxic bottom (e.g. Gilbert 2016)? Does it play a role in shallow lakes? Are there natural populations in which this trait is highly widespread, or even monomorphic? What are the consequences of short diapause for the formation of RE banks and for the genetic structure of such populations? An important step towards answering some of these questions might be the measurement of in situ hatching patterns of newly formed REs.

Acknowledgements Open access funding provided by University of Innsbruck and Medical University of Innsbruck.

Open Access This article is distributed under the terms of the Creative Commons Attribution 4.0 International License (http:// creativecommons.org/licenses/by/4.0/), which permits unrestricted use, distribution, and reproduction in any medium, provided you give appropriate credit to the original author(s) and the source, provide a link to the Creative Commons license, and indicate if changes were made.

\section{References}

Aparici, E., M. J. Carmona \& M. Serra, 1998. Sex allocation in haplodiploid cyclical parthenogens with density-dependent proportion of males. The American Naturalist 152(4): 652-657.

Aparici, E., M. J. Carmona \& M. Serra, 2002. Evidence for an even sex allocation in haplodiploid cyclical parthenogens. Journal of Evolutionary Biology 15: 65-73.

Becks, L. \& A. F. Agrawal, 2010. Higher rates of sex evolve in spatially heterogeneous environments. Nature 468(7320): 89-92.

Boschetti, C., F. Leasi \& C. Ricci, 2011. Developmental stages in diapausing eggs: an investigation across monogonont rotifer species. Hydrobiologia 662(1): 149-155.

Brendonck, L., 1996. Diapause, quiescence, hatching requirements: what we can learn from large freshwater branchiopods (Crustacea: Branchiopoda: Anostraca, Notostraca, Conchostraca). Hydrobiologia 320(1-3): 85-97.

Brendonck, L. \& L. De Meester, 2003. Egg banks in freshwater zooplankton: evolutionary and ecological archives in the sediment. Hydrobiologia 491(1-3): 65-84.
Campillo, S., E. M. Garcia-Roger, M. J. Carmona, A. Gomez \& M. Serra, 2009. Selection on life-history traits and genetic population divergence in rotifers. Journal of Evolutionary Biology 22(12): 2542-2553.

Carmona, M. J., N. Dimas-Flores, E. M. Garcia-Roger \& M. Serra, 2009. Selection of low investment in sex in a cyclically parthenogenetic rotifer. Journal of Evolutionary Biology 22(10): 1975-1983.

Denekamp, N. Y., M. A. Thorne, M. S. Clark, M. Kube, R. Reinhardt \& E. Lubzens, 2009. Discovering genes associated with dormancy in the monogonont rotifer Brachionus plicatilis. BMC Genomics 10(1): 108.

Denekamp, N. Y., R. Reinhardt, M. W. Albrecht, M. Drungowski, M. Kube \& E. Lubzens, 2011. The expression pattern of dormancy-associated genes in multiple life-history stages in the rotifer Brachionus plicatilis. Hydrobiologia 662(1): 51-63.

Garcia-Roger, E. M., M. J. Carmona \& M. Serra, 2005. Deterioration patterns in diapausing egg banks of Brachionus (Muller, 1786) rotifer species. Journal of Experimental Marine Biology and Ecology 314(2): 149-161.

Gilbert, J. J., 1974. Dormancy in rotifers. Transactions of the American Microscopy Society 93(4): 490-513.

Gilbert, J. J., 1989. Rotifera. In Adiyodi, K. G. \& R. G. Adiyodi (eds), Fertilization, development, and parental care. Wiley, Chichester.

Gilbert, J. J., 1995. Structure, development and induction of a new diapause stage in rotifers. Freshwater Biology 34 : 263-270.

Gilbert, J. J., 2001. Spine development in Brachionus quadridentatus from an Australian billabong: genetic variation and induction by Asplanchna. Hydrobiologia 446(1): 19-28.

GIlbert, J. J., 2016. Resting-egg hatching and early population development in rotifers: a review and a hypothesis for differences between shallow and deep waters. Hydrobiologia 186: 1-9.

Gilbert, J. J. \& M. C. Dieguez, 2010. Low crowding threshold for induction of sexual reproduction and diapause in a Patagonian rotifer. Freshwater Biology 55(8): 1705-1718.

Gilbert, J. J. \& D. K. Schreiber, 1998. Asexual diapause induced by food limitation in the rotifer Synchaeta pectinata. Ecology 79(4): 1371-1381.

Gilbert, J. J. \& T. Schroder, 2004. Rotifers from diapausing, fertilized eggs: unique features and emergence. Limnology and Oceanography 49(4): 1341-1354.

Hagiwara, A., N. Hoshi, F. Kawahara, K. Tominaga \& K. Hirayama, 1995. Resting eggs of the marine rotifer Brachionus plicatilis Müller: development, and effect of irradiation on hatching. Hydrobiologia 313-314(1): 223-229.

Hairston, N. G., 1996. Zooplankton egg banks as biotic reservoirs in changing environments. Limnology and Oceanography 41(5): 1087-1092.

Kilham, S. S., D. A. Kreeger, S. G. Lynn, C. E. Goulden \& L. Herrera, 1998. COMBO: a defined freshwater medium for algae and zooplankton. Hydrobiologia 377: 147-159.

Lewis, W. M., 1983. Interruption of synthesis as a cost of sex in small organisms. American Naturalist 121(6): 825-834.

Martínez-Ruiz, C. \& E. M. García-Roger, 2015. Being first increases the probability of long diapause in rotifer resting eggs. Hydrobiologia 745(1): 111-121. 
Pourriot, R. \& T. W. Snell, 1983. Resting eggs in rotifers. Hydrobiologia 104(1): 213-224.

Scheuerl, T. \& C. P. Stelzer, 2013. Patterns and dynamics of rapid local adaptation and sex in varying habitat types in rotifers. Ecology and Evolution 3(12): 4253-4264.

Schröder, T., 2005. Diapause in monogonont rotifers. Hydrobiologia 546: 291-306.

Schröder, T., S. Howard, M. L. Arroyo \& E. J. Walsh, 2007. Sexual reproduction and diapause of Hexarthra sp. (rotifera) in short-lived ponds in the Chihuahuan Desert. Freshwater Biology 52(6): 1033-1042.

Serra, M. \& T. W. Snell, 2009. Sex loss in monogonont rotifers. In Schön, I., K. Martens \& P. Van Dijk (eds), Lost sex. Springer, Berlin.

Stelzer, C. P., 2011. The cost of sex and competition between cyclical and obligate parthenogenetic rotifers. The American Naturalist 177(2): E43-E53.
Stelzer, C. P., 2015. Does the avoidance of sexual costs increase fitness in asexual invaders? Proceedings of the National Academy of Sciences USA 112(29): 8851-8858.

Stelzer, C.-P., J. Lehtonen, 2016. Diapause and maintenance of facultative sexual reproductive strategies. Philosophical Transactions of the Royal Society B 371: 20150536. doi:10.1098/rstb.2015.0536.

Storch, O., 1924. Die Eizellen der heterogonen Rädertiere: nebst allgemeinen Erörterungen über die Cytologie des Sexualvorganges und der Parthenogenese. Zool Jb Abt Anat 45: 309-404.

Wurdak, E. S., J. J. Gilbert \& R. Jagels, 1978. Fine structure of the resting eggs of the rotifers Brachionus calyciflorus and Asplanchna sieboldi. Transactions of the American Microscopical Society 97: 49-72. 